\title{
Quasiequilibrium time-domain susceptibility of semiconductor quantum wells
}

\author{
J. Javaloyes ${ }^{1, *}$ and S. Balle ${ }^{2, \dagger}$ \\ ${ }^{1}$ Department of Electronics and Electrical Engineering, University of Glasgow, Rankine Building, Oakfield Avenue, \\ Glasgow G12 8LT, United Kingdom \\ ${ }^{2}$ Institut Mediterrani d'Estudis Avançats, CSIC UIB, E-07071 Palma de Mallorca, Spain
}

(Received 2 February 2010; published 10 June 2010)

\begin{abstract}
We analyze the time-domain optical response of quantum well (QW) media in the quasiequilibrium approximation. The resulting macroscopic polarization can be expressed as a convolution integral that permits a simple and efficient numerical implementation for its use in dynamical modeling. As a practical example, the resulting polarization is used in conjunction with a travelling-wave model (TWM) for analyzing saturable absorption as a pulse propagates in a waveguide that incorporates an unpumped QW in its core. Frequency-selective saturable absorption and pulse distortion are observed.
\end{abstract}

DOI: 10.1103/PhysRevA.81.062505

PACS number(s): 42.55.Px, 78.66.-w

\section{INTRODUCTION}

The analysis of the dynamical properties of lasers requires a knowledge of the coupling between the active material and the optical field. The interaction between individual atoms and the electromagnetic radiation is at the origin of the development of quantum mechanics, and the interpretation of atomic spectra has been the object of a large wealth of work, e.g., cf. Refs. [1-5].

Within the semiclassical approach often invoked to model lasers, several authors have developed ways of treating the interaction between a quantized two-level or multilevel atom, with a formalism analogous to the vector description of spin states and the classical macroscopic electromagnetic field [6]. Such an approach provides the basis for successfully modeling gas and solid-state lasers. In these systems, the active medium can be described in an effective way as an ensemble of atoms or molecules with only two levels among which stimulated emission takes place $[7,8]$. In this approximation, the relevant variables for describing the active medium are the population inversion between these two levels, and the corresponding nonlinear polarization. Although the two-level atom model has been studied thoroughly and is usually assumed to be simple, subtle phenomena arise when high power $[9,10]$, multichromatic fields [11], or atomic motion [12] come into play.

Semiconductor media are conceptually similar to an ensemble of interacting atoms with different transition energies defined by the electronic band structure [13-15], but in spite of this conceptual similarity, a time-domain description of the macroscopic optical response of semiconductor materials is still an open problem. This is not only due to the complications arising from the band structure, but also because Fermi statistics imposes that the electronic states have different occupations depending on their energy. These two effects induce a large inhomogeneous broadening [16] that profoundly influences the dynamics of semiconductor lasers as compared to gas or solid state lasers. While the gain spectrum of a two-level atom is symmetric, even in the presence of

\footnotetext{
*julien.javaloyes@elec.gla.ac.uk

†salvador.balle@uib.es
}

Doppler inhomogeneous broadening, it is strongly asymmetric in semiconductors [13-15], resulting in a large degree of amplitude-phase coupling [17] that substantially affects the dynamical regimes and can even destroy any coherent emission in presence of feedback [18].

A direct approach to the interaction between a semiconductor material and an optical field is provided by the microscopic semiconductor Bloch equations [19] which consider the dynamics of each electronic state either including many-body effects [19-23] or neglecting them as in [13-15,24]. These microscopic theories describe each individual transition by the occupation of the initial and final electronic states, and the material polarization is obtained by superposing the contributions from each transition. A dynamical description of the lasing process then requires dealing with a large number of two-level like systems coupled among them by scattering processes ( $N$-body coupling) and by the optical field (mean-field coupling). This approach offers an excellent description of the material properties, but the complexity of such a description requires intensive numerical computations [25] which, in turn, limit their applicability for parametric studies of the dynamical scenarii in semiconductor lasers.

In order to bypass the complexity and high computational cost of microscopic theories, analytical approximations for the optical response of semiconductor media can be found in simple cases for both bulk $[26,27]$ and QW [28,29] semiconductor media. However, these results are known only in frequency domain and strictly valid only for a monochromatic field. For quasi-monochromatic fields, these analytical optical responses have been used in time-domain for studying the dynamics of the transverse modes in broad-area vertical-cavity surfaceemitting lasers (VCSELs) [30,31], the passive mode locking of external-cavity VCSELs $[32,33]$ or the multimode dynamics in conventional edge-emitting lasers [34,35]. Nevertheless, their application for studying intense and broadband time dependent optical fields is not straightforward.

A method for circumventing this problem was presented in [36], where a Padé approximation to the semiconductor susceptibility of $[28,29]$ was developed. Since the Padé approximant is a rational fraction of frequency, transformation to time domain is immediate, hereby allowing to explicitly determining an evolution equation for the macroscopic 
polarization of the active medium. This approach is similar to that in [37], where the optical response of the semiconductor medium determined from the semiconductor Bloch equations was fitted to the sum of several Lorentzians, each of them allowing for a two-level like description.

It must be noted, however, that the accuracy of the results based on this type of rational fits is expected to decrease either when increasing the optical bandwidth or when dealing with response functions that vary rapidly in frequency domain, like a saturable absorber (SA) close to its bandgap. In this case, the sharp transition-in frequency domain-from transparent to absorptive behavior is poorly described by any kind of fit based on polynomials or rational functions. It is worth noting that the two effects of broad-band dynamics and rapidly varying spectral features simultaneously occur in passively mode-locked lasers based on intracavity SA [38].

The large number of potential applications of short pulse sources in medicine, metrology and telecommunications [39] calls for a better understanding of the mode-locking problem and more generally the mechanisms by which modes in a semiconductor laser synchronize. However, the study of multimode dynamics faces a huge stiffness problem: while the multimode evolution of semiconductor lasers usually settles on asymptotic time scales of the order of hundreds of ns or even a $\mu \mathrm{s}$, the fastest time scale at work is typically a hundred of fs. Thus, the microscopic approach becomes too costly for attacking such regimes that span seven decades in time domain.

In this paper, we present an analytical time-domain optical response of a two-band semiconductor QW obtained in the quasiequilibrium limit. In spite of the approximations made, it yields realistic carrier dependent gain and index spectra, and it can be a useful tool for studying parametric dependencies at a marginally low computational cost. The resulting macroscopic polarization can be expressed as a convolution integral that permits a simple and efficient numerical implementation for its use in dynamical modeling. The validity and accuracy of this approach are tested by analyzing pulse propagation in a waveguide that incorporates an unpumped QW as a saturable absorber.

The paper is organized as follows. In Sec. II, we present the analytical results for the time-domain response. In Sec. III we discuss their numerical implementation within a TWM approach. In Sec. IV we perform several numerical experiments, e.g., frequency dependent absorption and strong nonlinear pulse shaping thereby demonstrating the physical effects that can be reproduced with our approach. Finally, in Sec. V we present the conclusions of our work.

\section{ANALYTICAL RESULTS}

\section{A. Direct time domain approach}

We consider a small volume of QW semiconductor material with linear dimensions quite smaller than a wavelength but yet encompassing many unit cells in the crystal. Assuming that there are only one conduction and one valence band, both parabolic with effective masses $m_{e}$ and $m_{h}$, respectively, the time-dependent intraband electron $\left[n_{k}(t)\right]$ and hole $\left[h_{k}(t)\right]$ distributions in the presence of an optical field $\mathcal{E}=E(t) e^{-i \Omega t}+$ c.c. are given, in the rotating-wave and dephasing-rate approximations, by [40]

$$
\begin{gathered}
\partial_{t} p_{k}=-\Gamma_{k} p_{k}-i g_{k}\left(n_{k}+h_{k}-1\right) E, \\
\partial_{t} n_{k}=-\frac{n_{k}-\bar{n}_{k}}{\tau_{e, k}}-i g_{k}\left(E^{\star} p_{k}-\text { c.c. }\right), \\
\partial_{t} h_{k}=-\frac{h_{k}-\bar{h}_{k}}{\tau_{h, k}}-i g_{k}\left(E^{\star} p_{k}-\text { c.c. }\right),
\end{gathered}
$$

where $\bar{n}_{k}$ and $\bar{h}_{k}$ are the stationary intraband distributions of electrons and holes, $p_{k}$ is the electron-hole coherence, $g_{k}$ is the coupling strength which represents the dipolar moment $d_{k}$ divided by $\hbar$, and $E(t)$ is the slowly varying amplitude of the optical field whose carrier frequency is $\Omega$. The relaxation toward stationarity of the electron and hole distributions is described in the simplest approximation of constant rates $\tau_{e, k}$ and $\tau_{h, k}$ toward local equilibrium [41], where the polarization dephasing rate reads $\gamma_{k}=\left(\tau_{e, k}^{-1}+\tau_{h, k}^{-1}\right) / 2$, and $\Gamma_{k}=\gamma_{k}+i\left(\omega_{k}-\Omega\right)$. $\hbar \omega_{k}=E_{\text {gap }}+\hbar^{2} k^{2} /\left(2 m_{r}\right)$ is the reduced energy of the electron-hole pair and $m_{r}$ is the reduced electron and hole mass. Since the optical carrier frequency can be freely chosen, we shall henceforth consider that $\Omega=E_{\text {gap }} / \hbar$.

The macroscopic optical polarization of the system, $\mathcal{P}(t)=$ $P(t) e^{-i \Omega t}+$ c.c. is given by the electron-hole coherence through

$$
P(t)=\frac{1}{V} \sum_{k} d_{k} p_{k}(t),
$$

where the summation runs over all electronic states, i.e., spin orientations and from $k=0$ up to $k_{m}$, the maximum wave vector in the first Brillouin zone of the crystal. Still, in order to obtain $P(t)$ we must integrate Eqs. (1)-(3), which do not possess a closed-form analytical solution.

In order to obtain the time dependent macroscopic polarization of the active medium, we define the saturation field of the two-level atom transition, $E_{\text {sat }}=\left(4 g_{k}^{2} \tau_{e} \tau_{h}\right)^{-1 / 2}$, and define a smallness parameter $\varepsilon=1 / E_{\text {sat }}$. We consider fields weak compared to $E_{\text {sat }}$, i.e., $E=\varepsilon \tilde{E}$ with $\tilde{E} \sim \mathcal{O}(1)$, and expand the polarization and carriers into the odd-even series [11] as

$$
\begin{aligned}
& p_{k}=\varepsilon p_{k}^{(1)}+\varepsilon^{3} p_{k}^{(3)}+\cdots, \\
& n_{k}=n_{k}^{(0)}+\varepsilon^{2} n_{k}^{(2)}+\cdots, \\
& h_{k}=h_{k}^{(0)}+\varepsilon^{2} h_{k}^{(2)}+\cdots .
\end{aligned}
$$

In this case, to the lowest order Eq. (1) reads

$$
\partial_{t} p_{k}=-\Gamma_{k} p_{k}-i g_{k}\left(n_{k}^{(0)}+h_{k}^{(0)}-1\right) E+\mathcal{O}\left(|E|^{2} E\right) .
$$

The higher order terms describe the effects of spectral hole burning and they are usually small in semiconductor lasers since the intraband relaxation rates are quite fast (of the order of $100 \mathrm{fs}$ ).

For time scales longer than the intraband relaxation times, the carriers have attained their stationary distributions, $\bar{n}_{k}$ and $\bar{h}_{k}$, respectively. Provided that we are not interested on such time scales and that the fields are not saturating, the quasiequilibrium approximation can be safely adopted. Within this approximation, the stationary intraband distributions 
of electrons and holes are given by the quasi-Fermi-Dirac distributions

$$
\begin{aligned}
& \bar{n}_{k}=\mathcal{F}\left[\frac{1}{k_{B} T}\left(\frac{\hbar k^{2}}{2 m_{e}}-\mu_{n}(t)\right)\right], \\
& \bar{h}_{k}=\mathcal{F}\left[\frac{1}{k_{B} T}\left(\frac{\hbar k^{2}}{2 m_{h}}-\mu_{h}(t)\right)\right],
\end{aligned}
$$

where $\mu(t)$ is the slowly time dependent Fermi level, $k_{B}$ is Boltzmann's constant, $T$ is temperature, and $\mathcal{F}(x)$ represents the Fermi-Dirac function.

Therefore, Eq. (8) can be formally solved as

$$
p_{k}(t)=\int_{0}^{\infty} d t^{\prime} R_{k}\left(t^{\prime}, t-t^{\prime}\right) E\left(t-t^{\prime}\right)+\mathcal{O}\left(|E|^{2} E\right),
$$

where we have defined the $k$-dependent convolution kernel

$$
R_{k}\left(t^{\prime}, t-t^{\prime}\right)=-i g_{k} e^{-\Gamma_{k} t^{\prime}}\left[\bar{n}_{k}\left(t-t^{\prime}\right)+\bar{h}_{k}\left(t-t^{\prime}\right)-1\right] .
$$

Hence, the quasiequilibrium macroscopic optical polarization is given by

$$
P(t)=\varepsilon_{0} \int_{0}^{\infty} d t^{\prime} \chi\left(t^{\prime}, t-t^{\prime}\right) E\left(t-t^{\prime}\right)+\mathcal{O}\left(|E|^{2} E\right),
$$

where the convolution kernel reads

$$
\chi\left(t^{\prime}, t-t^{\prime}\right)=\frac{1}{\varepsilon_{0} V} \sum_{k} d_{k} R_{k}\left(t^{\prime}, t-t^{\prime}\right) .
$$

It is worth recalling that Eq. (8) was the starting point in [29] for finding the quasiequilibrium frequency-dependent susceptibility $\tilde{\chi}(\omega, N)$ of the medium to a monochromatic field under the approximations of low temperature, charge neutrality and $k$-independent dipolar moment and intraband relaxation rates, i.e., $g_{k}=g$ and $\gamma_{k}=\gamma_{\perp}$. This yields

$$
\begin{aligned}
\tilde{\chi}(\omega, N)= & -\chi_{0}\left[2 \ln \left(1-\frac{D}{u+i}\right)\right. \\
& \left.-\ln \left(1-\frac{b}{u+i}\right)\right],
\end{aligned}
$$

where $\chi_{0}=m_{r} d^{2} /\left(\pi \varepsilon_{0} W \hbar^{2}\right), b=\hbar k_{m}^{2} /\left(2 m_{r} \gamma_{\perp}\right)$, and the carrier density and frequency were scaled as

$$
D=\frac{N}{N_{t}}, \quad N_{t}=\frac{m_{r} \gamma_{\perp}}{\pi W \hbar}, \quad u=\frac{\omega}{\gamma_{\perp}},
$$

with $W$ being the width of the of the QW and $N_{t}$ its transparency carrier density. In the absence of current injection, i.e., $N=0$, the susceptibility in Eq. (15) exhibits absorption for field frequencies between the bandgap, i.e., $\omega=0$, and that corresponding to the maximum span of the bands, i.e., $\Omega_{T}=\gamma_{\perp} b=\hbar k_{m}^{2} /\left(2 m_{r}\right)$. Outside this interval, the system is transparent as dictated by the lack of electronic states, and the transition from transparent to absorptive or viceversa occurs in a frequency interval spanning $\sim 6 \gamma_{\perp}$. When $N>0$ due to current injection, the system presents gain in the frequency interval between the gap and the transparency level defined by the Bernard-Duraffourg condition [42].
Performing the summation in Eq. (14) under the same assumptions as in [29], we find that the explicit form of the convolution kernel $\chi\left(t^{\prime}, t-t^{\prime}\right)$ reads

$$
\begin{aligned}
\chi\left(t^{\prime}, t-t^{\prime}\right) & =\chi_{0} e^{-\gamma_{\perp} t^{\prime}} \frac{2 e^{-i \gamma_{\perp} D\left(t-t^{\prime}\right) t^{\prime}}-1-e^{-i \gamma_{\perp} b t^{\prime}}}{t^{\prime}}, \\
& \equiv \chi\left[t^{\prime}, N\left(t-t^{\prime}\right)\right] .
\end{aligned}
$$

\section{B. Discussion}

The convolution kernel in Eq. (17) determines the timedomain optical response of the semiconductor material in the quasiequilibrium approximation. The constraints imposed to the maximum field energies and the minimum pulse widths define the limits of applicability of our approach.

Notice however that these restriction are not too severe since in most situations, typical pulse widths in semiconductor lasers are of the order of the picosecond, i.e., longer than the intraband time, and possess modest energies. For definiteness, assuming $\tau_{e}=160 \mathrm{fs}, \tau_{h}=100 \mathrm{fs}$ and $d_{k}=4.7 \times 10^{-29} \mathrm{Cm}$, which are typical for In-Ga-As-P lasers, one obtains a saturation field $E_{\text {sat }}=8.9 \times 10^{6} \mathrm{Vm}^{-1}$. For a typical effective mode area $S_{m} \sim 1 \mu \mathrm{m}^{2}$ and as-cleaved facets (reflectivity $r \sim 0.55$ ), the power per facet emitted at saturation corresponds to $P_{\text {out }}=\left(1-r^{2}\right) \epsilon_{0} c n^{2}\left|E_{\text {sat }}\right|^{2} S_{m} / n_{g} \sim 500 \mathrm{~mW}$. In the case of a mode-locked laser operating at a repetition frequency $f_{\text {rep }} \sim 50 \mathrm{GHz}$, this would correspond to pulse energies $E_{\text {pulse }} \sim 10 \mathrm{pJ}$. Therefore, the quasiequilibrium approximation still holds even for ps pulses provided that their energy is not too high.

In addition, before discussing the practical aspects of our approach it is worth examining the convolution kernel $\chi\left(t^{\prime}, t-t^{\prime}\right)$ in some detail.

(1) The convolution kernel depends only on the past values of the carrier density in accordance with physical intuition. It depends both on the retarded time argument $t^{\prime}$ and on the instantaneous value of the time instant $t$. This simply reflects the fact that the value of the gain and of the refractive index depends on the current value of the time evolving population inversion $N\left(t-t^{\prime}\right)$. Furthermore, this $N$-dependence is non linear. As such $\chi\left(t^{\prime}, t-t^{\prime}\right)$ is a causal, nonlinear and time variant filter.

(2) Equation (17) can be obtained by Fourier transform of the frequency and carrier-dependent susceptibility defined by Eq. (15), although the procedure is quite involved and it is unclear at which times the contributions of the carrier density have to be considered in the convolution kernel. Indeed, Eq. (15) was derived under the monochromatic assumption for which the carrier density is time independent. The approach taken here is more direct and clearer in what concerns the time dependence of the contribution of the carriers.

(3) The convolution kernel $\chi\left(t^{\prime}, t-t^{\prime}\right)$ is well behaved at $t^{\prime}=0$ since

$$
\chi\left[t^{\prime}, N\left(t-t^{\prime}\right)\right] \underset{t^{\prime} \rightarrow 0^{+}}{\longrightarrow} i \chi_{0} \gamma_{\perp}\left[b-2 N(t) / N_{t}\right] .
$$

(4) The convolution kernel rapidly decays to zero with the characteristic time scale $\gamma_{\perp}^{-1} \sim 100$ fs, i.e., the inverse of the polarization decay rate. This characteristic is very convenient for numerical purposes, since it implies that in 
order to determine the macroscopic polarization only field and carrier contributions within the last 1 ps, say, must be considered.

(5) The inspection of Eqs. (13) and (17) calls for a helpful approximation. The kernel of integration $\chi$ being nonzero from $t^{\prime}=0$ to $t^{\prime} \sim 3 \gamma_{\perp}^{-1}$, i.e., a few hundred femtoseconds it is possible to assume that the carrier density $N(z, t)$ does not change appreciably over this time interval. This suggests to perform a first order Taylor expansion of Eq. (17)

$$
\chi\left[t^{\prime}, N\left(t-t^{\prime}\right)\right] \sim \chi\left[t^{\prime}, N(t)\right]+t^{\prime} \dot{N}(t) \frac{\partial \chi}{\partial N}+\cdots .
$$

Typically, QW lasers operate close to transparency, i.e., $N \sim \mathcal{O}\left(N_{t}\right)$, and the carrier density decays on a time scale of $\gamma_{\|}^{-1} \sim 1$ ns, hence the relative error incurred when the first order correction is neglected is

$$
e=\left|t^{\prime} \dot{N}(t) \frac{\partial \chi}{\partial N} / \chi\left[t^{\prime}, N(t)\right]\right| \sim \gamma_{\|} \gamma_{\perp}^{-1} \sim 10^{-4} .
$$

Thus, provided that the time evolution of the carrier density over a time $\gamma_{\perp}^{-1}$ remains small, we can safely replace $N\left(t-t^{\prime}\right) \rightarrow N(t)$ in Eqs. (13) and (17). The above statement can only be violated if one considers the dynamics of highly energetic sub-picosecond pulses where the strongly nonlinear stimulated emission can modify appreciably the carrier density within a time interval of a few hundred femtoseconds. Notice however that in this case not only our first order expansion in the field amplitude in Eq. (13) is invalid, but also that the quasiequilibrium approximation does no longer hold. We conclude that neglecting the retarded time argument in Eqs. (13) and (17) is consistent with neglecting the $\mathcal{O}\left(|E|^{2} E\right)$ terms in Eq. (13).

(6) In order to derive Eqs. (15) and (17) we neglected the $k$-dependence of the dipole moment $g_{k}$ and of the polarization dephasing rate $\gamma_{k}$. It is however known that the dipolar moment and the dephasing rate are decreasing and increasing functions of the energy [19], respectively. We note that these effects can be incorporated in our approach but at the price of a more elaborated convolution kernel and a less intuitive interpretation. One of the dominant effects of this $k$-dependence is to decrease of the light-matter interaction for high energies thereby inducing an effectively smaller energy span of the bands: the strongly blue detuned transitions possess a lower interaction with the field and a faster decay rate so that their contributions to the optical response become less important.

\section{IMPLEMENTATION}

The macroscopic polarization of the system given by Eq. (13) provides the source term that has to be considered when analyzing the dynamics of the optical field. For this purpose, different modeling strategies have been developed, the most widely used being - in order of increasing numerical difficulty - the rate equation (RE) description, the traveling wave modeling (TWM) [43] and the finite differences in time-domain (FDTD) [44] method.

RE-based descriptions do not explicitly consider the spatial variations. The field is expanded into cavity modes, and the spatial dependence of all variables is removed by projection onto these modes. In this case, Eq. (13) can be directly used, with the convention that the field $E$ in Eq. (13) has to be understood as the amplitude of the sought optical mode, $P$ as the projection of the total polarization onto this cavity mode, and $N$ as the spatial average of the carrier density.

Conversely, the FDTD method is based on the direct resolution of Maxwell's equations, so the local polarization of the active medium has to be provided. In this case, Eq. (13) can also be directly used as the local constitutive relation of the active medium, with just one trivial modification: note that the FDTD method deals with the total field, polarization, and carrier density - which exhibit temporal and spatial variations of the order of the optical frequency and the wavelength, respectively-hence a rotating exponential $\exp (i \Omega r)$ must multiply Eq. (17).

Instead, the application of Eq. (13) to the case of a TWM is not direct, because the TWM is based on the slowly varying approximation (SVA) and it involves decomposing the field into forward and backward propagating waves and the carrier density into slow and fast spatially evolving components. Therefore in Sec. III A we briefly recall the TWM approach and discuss how to exploit Eq. (13) in this context.

In Sec. III B we discuss the numerical algorithm used to implement the convolution integral that determines the macroscopic polarization from the past values of the local field and carrier density. The temporal discretization inherent to numerical integration imposes that the convolution has to be performed from a sampled signal, which induces several kind of errors that we discuss in Sec. III C. In addition, the large energy span of the semiconductor QW bands, represented by the $b$ factor in Eqs. (15) and (17), proves to be the main source of stiffness in the convolution method. We discuss how this problem can be overcome in Sec. III D.

\section{A. General form of the TWM}

In this subsection we briefly recall the general form of TWMs and we discuss how to implement the convolution integral defining the macroscopic polarization in this context. We refer the reader to $[36,45]$ and references therein for more details on the traveling wave modeling approach.

The evolution of the normalized slowly-varying amplitudes of the forward and backward waves $E_{ \pm}$reads

$$
\left(\partial_{t} \pm \partial_{z}\right) E_{ \pm}(z, t)=i \frac{q_{w} \Gamma \Omega L}{2 \epsilon_{0} n_{g} c} P_{ \pm}(z, t)-\alpha_{i} E_{ \pm}(z, t)
$$

where $\alpha_{i}$ are the internal losses of the system scaled to length of the cavity $L, \Gamma$ is the confinement factor of the optical field to one $\mathrm{QW}, q_{w}$ the number of $\mathrm{QW}$, and $n_{g}$ the group index. Space and time are scaled to the cavity length $L$ and transit time $\tau_{c}=n_{g} L / c$, respectively. $P_{ \pm}$are the projection of the total polarization at $(z, t)$ onto the forward and backward propagation directions. They are obtained by a coarse graining procedure by averaging the polarization over a few wavelengths [46].

The total carrier density is decomposed as $N(z, t)=$ $N_{0}(z, t)+\left[N_{+2}(z, t) e^{2 i q_{0} z}+N_{-2}(z, t) e^{-2 i q_{0} z}\right]$, where $N_{0}(z, t)$ is the quasi-homogeneous component, $N_{ \pm 2}(z, t)$ is the complex amplitude of the (weak) grating component arising from 
standing wave effects in the system and $q_{0}=\left(2 \pi n_{g}\right) / \lambda$ is the optical carrier wave vector. Their evolution is given by

$$
\begin{aligned}
& \tau_{c}^{-1} \partial_{t} N_{0}(z, t)= \frac{I}{e q_{w} V_{a}}-R\left(N_{0}\right) \\
&-\frac{i}{\hbar}\left(P_{+} E_{+}^{\star}+P_{-} E_{-}^{\star}-\text { c.c. }\right), \\
& \tau_{c}^{-1} \partial_{t} N_{ \pm 2}(z, t)=-\left[R^{\prime}\left(N_{0}\right)+4 \mathcal{D} q_{0}^{2}\right] N_{ \pm 2} \\
&-\frac{i}{\hbar}\left(P_{ \pm} E_{\mp}^{\star}-E_{ \pm} P_{\mp}^{\star}\right),
\end{aligned}
$$

where $I$ is the current injected into a QW whose volume is $V_{a}, e$ is the electron charge, and $R$ stands for the recombination term that includes the usual nonradiative, bimolecular, and Auger recombination terms, as such $R(N)=A N+B N^{2}+C N^{3}$, $R^{\prime}(N)=d R / d N$ and the ambipolar diffusion coefficient is $\mathcal{D}$.

The closure is made by linking the counter-propagating waves $E_{ \pm}(z, t)$ to the spatially and time dependent active material. From Eq. (13) we have that

$$
\begin{aligned}
P^{ \pm}(z, t)= & \varepsilon_{0} \int_{0}^{\infty} d t^{\prime}\left\{\chi\left[t^{\prime}, N_{0}\left(z, t-t^{\prime}\right)\right] E^{ \pm}\left(z, t-t^{\prime}\right)\right. \\
& \left.+\chi_{N}\left[t^{\prime}, N_{0}\left(z, t-t^{\prime}\right)\right] N_{ \pm 2}\left(z, t-t^{\prime}\right) E^{\mp}\left(z, t-t^{\prime}\right)\right\},
\end{aligned}
$$

with $\chi\left[t^{\prime}, N\left(t-t^{\prime}\right)\right]$ defined in Eq. (17) and

$$
\chi_{N}\left(t^{\prime}, N\right) \equiv \frac{\partial \chi\left(t^{\prime}, N\right)}{\partial N}=-\frac{2 i \gamma_{\perp}}{N_{t}} e^{-\gamma_{\perp}(1+i D) t^{\prime}} .
$$

Upon inspection of Eq. (24) one notices that the counterpropagating waves $E^{ \pm}(z, t)$ couple through the grating induced in the carrier density $N_{ \pm 2}(z, t)$, the so-called spatial hole burning. For all purposes, one shall only keep track of $N_{+2}(z, t)$ since $N_{-2}(z, t)=N_{+2}^{\star}(z, t)$.

We recall that the field amplitudes $E^{ \pm}$and the polarization waves $P^{ \pm}$are in SI units, i.e., $\mathrm{Vm}^{-1}$ and $\mathrm{CVm}^{2}$, respectively. The conversion to optical energy (in Joules) is achieved by integrating the optical power (in Watts) $\epsilon_{0} c\left|E^{ \pm}\right|^{2} S_{m} / n_{g}$ over time where $S_{m}$ is the surface of the mode.

However, since our TWM is better described in dimensionless spatial and temporal units we shall give some order of magnitude of the relevant scales. By assuming a cavity length of $1.04 \mathrm{~mm}$ and an group index of refraction of $n_{g}=3.6$, we obtain a single trip in the cavity of $\tau_{c}=$ 12.5 ps. A typical spatial discretization requires $M=400$ mesh points, which in turns fixes the temporal discretization of the field time evolution by the Courant-Friedrichs-Lewy (CFL) condition [47]. In our notation both the temporal and spatial discretizations read $h=1 / M$ which corresponds to a spatial step of $h L=2.6 \mu \mathrm{m}$ and a temporal step of $h \tau_{c}=$ $31.25 \mathrm{fs}$. By choosing a typical value of an intraband relaxation time of $\gamma_{\perp}^{-1}=125$ fs one obtains a scaled decay rate of the polarization of $\tilde{\gamma}_{\perp}=\tau_{c} \gamma_{\perp}=100$, which in turns gives a scaled product of the time step and of the polarization decay rate $\tilde{\gamma}_{\perp} h=0.25$.

To conclude, we refer the reader to [48] where the discussion of how to impose proper boundary conditions in the TWM can be found. It is also worth to mention that the
TWM method of integration described in [48] is second order accurate, i.e., exact up to $\mathcal{O}\left(h^{3}\right)$.

\section{B. Convolution algorithm}

We assume that time is discretized with step $h$, so at time $t_{n}=n h$ Eq. (13) can be expressed as

$$
\begin{aligned}
P\left(t_{n}\right) & =\varepsilon_{0} \int_{0}^{\infty} d t^{\prime} \chi\left[t^{\prime}, N\left(t_{n}-t^{\prime}\right)\right] E\left(t_{n}-t^{\prime}\right), \\
& \approx \varepsilon_{0} \int_{0}^{K h} d t^{\prime} \chi\left[t^{\prime}, N\left(t_{n}-t^{\prime}\right)\right] E\left(t_{n}-t^{\prime}\right), \\
& =\varepsilon_{0} \sum_{m=0}^{K-1} J_{n m},
\end{aligned}
$$

where we have assumed that the convolution integral can be cut at a long enough delay $t_{K}=K h$. Moreover, we have defined

$$
J_{n m}=\int_{m h}^{(m+1) h} d t^{\prime} \chi\left[t^{\prime}, N\left(t_{n}-t^{\prime}\right)\right] E\left(t_{n}-t^{\prime}\right),
$$

which represents the contribution of the state of the system $m$ time steps ago to the present macroscopic polarization. Using the trapezoidal approximation to the integral, up to third order in $h$ we have that

$$
J_{n m} \simeq \frac{h}{2}\left(\chi_{n, m} E_{n-m}+\chi_{n, m+1} E_{n-m-1}\right),
$$

where $E_{n}=E\left(t_{n}\right)$ and $\chi_{n, m}=\chi\left[m h, N\left(t_{n-m}\right)\right]$. Notice that this second order approximation to the integral is consistent with the algorithm of integration of our TWM [48]. Hence we can finally express the macroscopic polarization at time $t_{n}$ as

$$
\frac{P_{n}}{\varepsilon_{0} \chi_{0}}=\sum_{m=1}^{K-1} q_{n, m} E_{n-m}+i \frac{h \tilde{\gamma_{\perp}}}{2} E_{n}\left(b-2 D_{n}\right),
$$

where

$$
q_{n, m}=e^{-\tilde{\gamma}_{\perp} h m} \frac{2 e^{-i D_{n-m} \tilde{\gamma}_{\perp} m h}-e^{-i b \tilde{\gamma}_{\perp} m h}-1}{m} .
$$

In Eq. (29), the second term represents the contribution of the first exterior point $(m=0)$ and we have considered that the contribution of the last exterior point is vanishingly small due to the decreasing exponential, i.e., $q_{n, K}=0$.

In the case where one neglects the delayed argument in the carrier density, this expression allows for an efficient numerical implementation as it can be streamlined as

$$
\begin{gathered}
q_{n, m}=\left[2 k_{m}-\left(l_{m}+r_{m}\right)\right] / m, \\
k_{m}=\kappa k_{m-1}, \\
l_{m}=\lambda l_{m-1}, \\
r_{m}=\rho r_{m-1},
\end{gathered}
$$

with $\kappa=e^{-\tilde{\gamma} \perp h\left(1+i D_{n}\right)}, \lambda=e^{-\tilde{\gamma_{\perp} h(1+i b)}}, \rho=e^{-\tilde{\gamma_{\perp} h}}$, and with the initial conditions $k_{0}=l_{0}=r_{0}=1$. Therefore, besides the cost of calculating the three complex exponentials $\kappa, \lambda$ and $\rho$, only three complex multiplications and one division are needed for each iteration of $m$. This implementation is versatile, although not the fastest since $l_{m} / m$ and $\rho_{m} / m$ are time independent quantities that can be evaluated once and for all. The only important quantities that cannot be tabulated 
beforehand are the $k_{m} / m$ cutting the cost to one initial complex exponential as well as one complex multiplication and division for each value of $m$.

To conclude, the same numerical algorithm can be used in conjunction with a TWM. In this case the discretized version of Eq. (24) reads

$$
\begin{aligned}
\frac{P_{n}^{ \pm}}{\varepsilon_{0} \chi_{0}}= & \sum_{m=1}^{K-1} q_{n, m} E_{n-m}^{ \pm}+i \frac{h \tilde{\gamma}_{\perp}}{2} E_{n}^{ \pm}\left(b-2 D_{n}^{0}\right) \\
& -2 i \tilde{\gamma}_{\perp} h\left[\sum_{m=0}^{K-1} D_{n-m}^{ \pm 2}\left(k_{n, m} E_{n-m}^{\mp}-\frac{E_{n}^{\mp}}{2}\right)\right],
\end{aligned}
$$

where $q_{n, m}$ is given by Eq. (30) with $D^{0}$ instead of $D$, and $k_{n, m}=e^{-i \tilde{\gamma}_{\perp} m h D_{n-m}^{0}}$.

\section{Numerical errors}

The numerical implementation of the convolution integral according to the algorithm above presents some computational problems that are worth discussing.

The most obvious error steams from the fact that the integral in Eq. (13) should span from $t_{\min }^{\prime}=0$ to $t_{\max }^{\prime}=\infty$. This poses the problem that a possibly infinite segment of the past history of the field has to be kept at each spatial position. However, convergence is reached fairly quickly in our case due to the decaying exponential in Eq. (17). The results are well within the convergence region if one uses $t_{K}=6 \gamma_{\perp}^{-1}$ in Eq. (26) and therefore $K$ is the nearest integer to $6 /\left(h \gamma_{\perp}\right)$. This typically corresponds to a time of the order of the picosecond which conveys a typical memory segment of $K=20$ past sampled values of the fields and carrier densities. Thus, the memory cost is marginal and the computing cost is light. An efficient way to store this past information is to use a circular array at each point of the spatial grid where for each time step the oldest value is replaced by the newest one.

A second, quite more subtle problem arises from the discrete nature of numerical integration. While the convolution integral in Eq. (13) determining the macroscopic polarization involves continuous time functions, its numerical implementation in Eqs. (29) and (32) relies on quantities that are sampled with a time interval $h$. First, the sampling rate $h$ has to be smaller than the inverse of the polarization decay time $\tilde{\gamma}_{\perp}^{-1}$ for numerical stability. Second, the sampling introduces a Nyquist cut-off frequency $\Omega_{N}=\pi / h$, hence the numerical implementation has to automatically present some aliasing. Clearly, the finer the discretization, the larger the cut-off frequency, but at the price of an increased computation time.

In order to test the numerical convolution algorithm, we present in Fig. 1 the comparison between the exact and the numerical susceptibilities of the medium for a monochromatic field at different frequencies $\omega$, in the case where $b=10$. The scaled numerical susceptibility (dashed lines) determined as $P_{n} /\left(\varepsilon_{0} \chi_{0} E_{n} e^{i \omega n h}\right)$ from Eq. (29) has been obtained with quite a large value of the product $\tilde{\gamma}_{\perp} h=0.25$ but already represents a very good approximation to the exact susceptibility $\tilde{\chi}(\omega, N) / \chi_{0}$ (solid lines) defined by Eq. (15) over the central frequency interval for both low and high carrier densities, i.e., $N=0$ and $N=3 N_{t}$, respectively. However, one clearly sees a discrepancy for the reddest and bluest
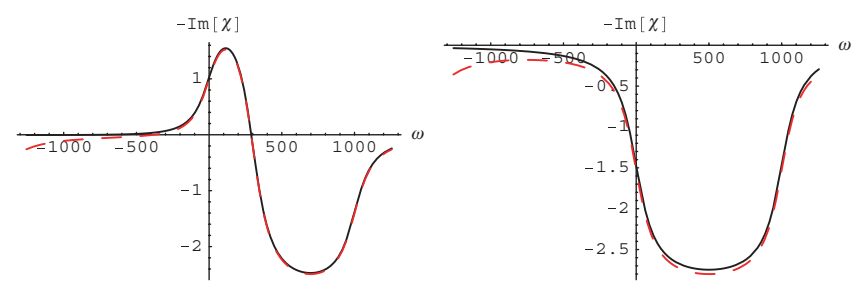

FIG. 1. (Color online) Analytical (black) and effective imaginary parts susceptibilities (red dashes) obtained from numerical convolution for fields at different frequencies $\omega$ for $\tilde{\gamma}_{\perp}=100$ and $b=10$. Left (right) panel corresponds to $N=3 N_{t}(N=0)$.

frequencies close to the Nyquist border. The reason of the this deformation is immediately clear if one looks at the same graph over a broader frequency range as in Fig. 2: we obtain an aliased, or periodized, susceptibility, the period being twice the Nyquist frequency. The effective susceptibility at the reddest frequency $\omega=-\Omega_{N}$ has to be equal to the effective susceptibility at the bluest frequency $u=\Omega_{N}$ since these two frequencies are indistinguishable due to aliasing. In our case, we have taken $h=1 / 400$, therefore the cut-off frequency is $\Omega_{N} \sim 1256$ while the top of the band where the blue transition to reabsorption occurs is located at $\Omega_{T}=\tilde{\gamma}_{\perp} b=1000$.

For a two-level atom, this problem would be less stringent since the shape of the gain is symmetric with respect to its peak value, therefore the bluest and reddest values are identical and only a slight distortion in the antisymmetric index of refraction should be expected. Instead, semiconductor media are transparent at the reddest frequencies and absorptive at the bluest ones, hence a deformation of the absorption/gain spectrum occurs.

This problem is readily overcome by using a sufficiently small value of $h$ in such a way that the dynamics expected in the central frequency region is not affected by the deformations occurring close to the Nyquist border and by choosing a cut-off $\Omega_{N}$ larger than $\Omega_{T}$.

\section{Stiffness and fidelity}

Inspection of Eq. (30) reveals that the term $e^{-i m \tilde{\gamma_{\perp}} b h}$ poses the most severe restrictions to the choice of $h$ : in principle, a faithful discretization of the numerical convolution kernel does not only require small values of $\tilde{\gamma}_{\perp} h$ but the more stringent condition $\tilde{\gamma}_{\perp} b h<\pi$ hence the large values of $\tilde{\gamma}_{\perp}$

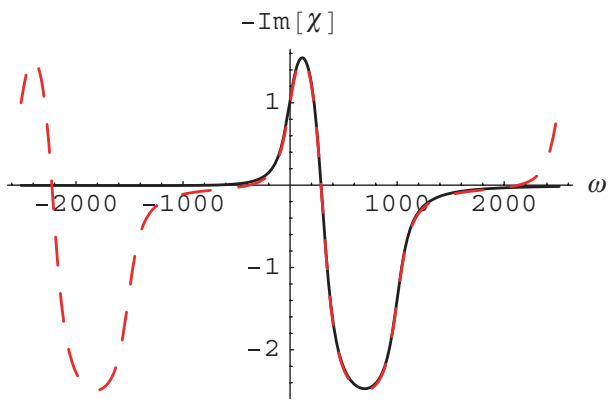

FIG. 2. (Color online) Same as Fig. 1 but extending the frequency interval to twice the Nyquist frequency. Aliasing in the numerical convolution clearly manifests as a periodization of the susceptibility. 


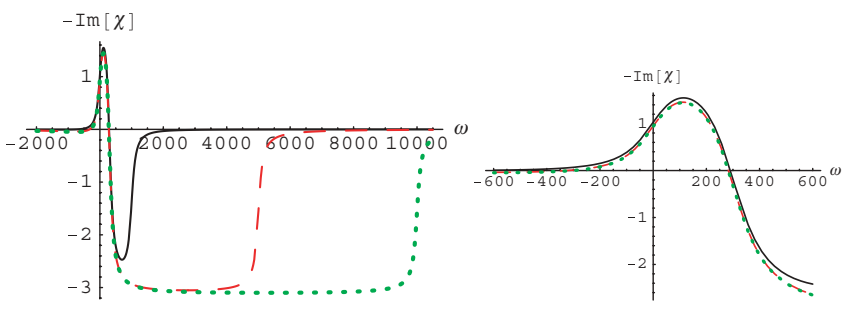

FIG. 3. (Color online) Left: Imaginary part of the susceptibility obtained from Eq. (15) for $b=10$ (black line), $b=50$ (red dashes), and $b=100$ (green dots). The parameters are $\tilde{\gamma}_{\perp}=100$ and $N=$ $3 N_{t}$. Right: Zoom of the normalized gain spectrum close to the gap region. In this spectral range the three curves almost coincide in spite of the different values of $b$.

and $b$ in semiconductor lasers would lead to extremely small and unpractical time steps.

In order to overcome this difficulty, it is worth reexamining the role of $b$ in the dynamics of the system. In frequency domain, $b$ represents the interval of photon energies above the gap-normalized to $\gamma_{\perp}$-over which optical transitions can occur, and bigger values of $b$ simply allow for transitions with higher energy (see Fig. 3 left panel). In addition, $b$ also determines the refractive index of the QW system (see Fig. 4 right panel), but in the frequency interval close to the bandgap, it does so in a trivial way, just adding a constant contribution that does not modify the effective $\alpha$ factor.

In addition, note that although unpractically small time steps would be required in order to describe dynamics over the large spectral region of absorption in the QW material, in most cases we are interested only in dynamics occurring in the spectral region close to the bandgap of the QW, where gain and saturable absorption occur. From a numerical point of view, the time step $h$ has to be chosen in such a way that it provides a faithful representation of such dynamics, hence much longer time steps could be used.

The above facts suggest that, since we are interested in the dynamics of fields whose spectral contents is close to the bandgap, we can artificially reduce the value of $b$ without affecting the amplification or attenuation in the spectral region of interest (see Fig. 3 right panel). The accompanying reduction in refractive index is not relevant: on one hand, this constant contribution can be absorbed into the propagation constant thereby renormalizing the optical length of the cavity; on the other, it has to be recalled that the actual refractive index to be considered in the active region is the difference
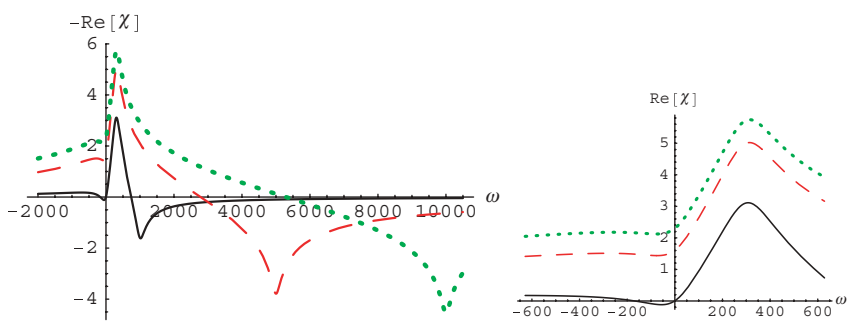

FIG. 4. (Color online) Same as in Fig. 3 but for the real part of the susceptibility. Notice that, in the spectral range of interest around the bandgap (right panel), the three curves are simply shifted upward as $b$ increases. between the refractive index of the active region and that of the nucleus of the waveguide (see Eq. (17) in [36]). Therefore, even if the actual semiconductor band is very wide as corresponding to $b \sim 10^{3}$, a smaller value of $b \sim 10-50$ can provide us with a good description of the dynamics close to the band edge; in fact, $b=10$ corresponds to an absorption band of $\Delta v=b \gamma_{\perp} /(2 \pi)=13 \mathrm{THz}-$ i.e., about $100 \mathrm{~nm}$ at $\lambda \sim 1.55 \mu \mathrm{m}$ - which is much broader than most optical fields. In addition, it is worth recalling that - as discussed in Sec. II B 7 -absorption at high frequency is overestimated due to the decrease of dipole moment and the increase in dephasing rate with energy, hereby lowering the effective values of $b$. For all these reasons, we can use a lower value of $b$ which allows us to safely use much longer time steps $h$ provided that the condition $\tilde{\gamma}_{\perp} b h<\pi$ holds.

In summary, the main restriction on the time step $h$ does not stem from the fast evolution of the polarization as compared to the field and carrier density, this requirement being controlled by the scaled quantity $\tilde{\gamma}_{\perp} h$. Instead, it comes from the effective value of $b$ that can be used in order to have the resulting dynamics in a frequency region well contained in the middle of the numerical bandwidth, far from the numerical artifacts at the reddest and the bluest frequencies. This last condition is controlled by using a sufficiently large value of $b$ and by fulfilling the more stringent requirement $\tilde{\gamma}_{\perp} b h<\pi$, which ultimately controls the stiffness of the system.

\section{NUMERICAL TESTS}

In order to evaluate the potential of our method, in this section we analyze different situations encountered when an optical pulse is injected into a waveguide containing an unpumped QW in its core. As already commented, this case, which corresponds to propagation into a SA, can hardly be reproduced with a Padé approximant due to the sharp frequency dependence of the absorption, see for instance Fig. 1.

We consider a cavity of length $1.04 \mathrm{~mm}$ and effective group index $n_{g}=3.6$ with both facets being antireflection coated $\left(r=10^{-6}\right)$. Note that this cavity configuration with extremely small reflectivities represents quite a special case of our model. We have chosen it because it allows for simpler physical understanding of the numerical results since in this limit, when one injects a pulse from the left facet into the forward direction, the reflected component $E_{-}(z, t)$ is always very small and as such $P_{-}(z, t) \approx 0 \approx N_{2}(z, t)$, hereby permitting to compare the results of the bidirectional TWM with the intuitive picture of unidirectional propagation. We remark however that that this special configuration serves only as an illustration of the capabilities of our approach for reproducing the strong spectral filtering of a SA. See for instance [45] for a more general use of our model in a two-section Fabry-Pérot mode-locked laser with cleaved facets.

The active material consists of five QWs, i.e., $q_{w}=5$ of width $W=6 \mathrm{~nm}$, the dipole moment is $d=4.7 \times$ $10^{-29} \mathrm{Cm}$, the reduced mass is $m_{r}=5 \times 10^{-32} \mathrm{~kg}$, the confinement factor is $\Gamma=1 \%$, and the electron and hole relaxation times are $\tau_{e}=160 \mathrm{fs}$ and $\tau_{h}=100 \mathrm{fs}$, respectively. The saturation field is $E_{\mathrm{sat}}=8.9 \times 10^{6} \mathrm{Vm}^{-1}$, and assuming a mode spot size of $1 \mu \mathrm{m}^{2}$ we obtain a saturation power of 
$\sim 750 \mathrm{~mW}$. We deduce that the gain bandwidth is $\gamma_{\perp}^{-1}=125 \mathrm{fs}$, the transparency carrier density $N_{t}=2.1 \times 10^{23} \mathrm{~m}^{-3}$ and that the maximum modal absorption in the unpumped $\mathrm{QW}$ amounts to $q_{w} \Gamma \pi \chi_{0} \Omega /\left(c n_{g}\right)=120 \mathrm{~cm}^{-1}$.

Due to the very large absorption of the QW, internal losses are not playing any role in the numerical tests that follow, therefore we assume for the sake of simplicity $\alpha_{i}=0$. For the same reasons, due to the low carrier density present in the SA we neglect as well bimolecular and Auger recombination coefficients. We assume that we are in the case of a slow SA, as such the recovery time $A^{-1}$ is $1 \mathrm{~ns}$.

The cavity is discretized with $M=400$ spatial points. We monitor the field output through the right facet of the slab, while an optical pulse can be injected through the opposite, left facet.

Finally, in order to simulate spontaneous emission noise we add to the polarization waves $P_{ \pm}$, at each time step and at each spatial point, a stochastic component consisting of Gaussian white noise of zero mean and variance $\beta=10^{-2}$ which we assume for the sake of simplicity to be carrier density independent. The noise was generated by the Box-Muller method [49] and a Mersenne twister [50].

\section{A. Noise emission spectrum}

We produced several noise spectrum by the following method. The simulation time is $12.5 \mathrm{~ns}$ which correspond to $4 \times 10^{5}$ samples used for the Fourier transform. The memory segment is composed of $K=25$ points. Thorough this section, we checked that we obtained identical results with $K=50$. The optical spectra were smoothed by averaging over $M$ channels. We therefore cut the $M$ first channels on the red and blue side that cannot be averaged. We started by producing a noise spectrum of a passive cavity, which is achieved by enforcing $\chi_{0}=0$, and a second noise spectrum with $\chi_{0}=2$. In this latter case, since there is no electrical pumping in the cavity, i.e., $J=0$ the population inversion is $N(z, t)=0$, up to small statistical fluctuations.

The red (lower) line in Fig. 5 presents the optical spectrum of the emission through the right facet while for the sake of comparison, we also show the noise spectrum of the transparent waveguide as a black (upper) line. We can see that transparent waveguide yields a flat noise spectrum,

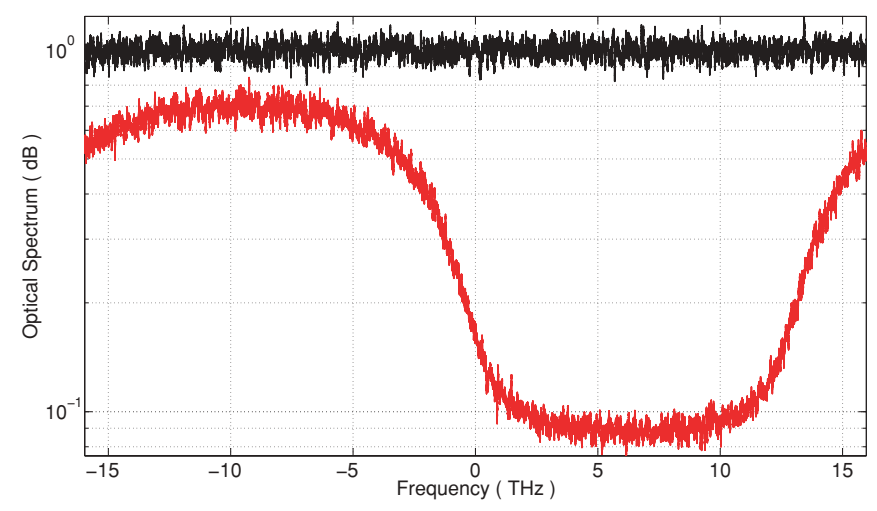

FIG. 5. (Color online) Optical spectra of a transparent waveguide [black (upper)] and of a waveguide with an unpumped QW in its core [red (lower)]. while the results for the QW system nicely reproduce the absorption characteristics of the unpumped QW, in particular the band edge of the semiconductor. However, one can also notice the extra residual absorption below the band edge due to the aliasing artifacts discussed earlier. It must be noted that at the band-edge frequency, where the absorption coefficient of the empty QW should be exactly 1/2 of the maximum value, the spectral density of the noise is not midway between the maximum and minimum noise densities. This is a simple consequence of the fact that even in the linear regime, the resulting spectral density is not a linear but an exponentially decreasing function of the slab length times the absorption coefficient.

\section{B. Small signal multimode response}

When a pulse is injected into the waveguide with the unpumped QW, the output field has to present frequencyselective absorption with saturable effects appearing as the input power is increased. In order to study the effects of this frequency-selective saturable absorption, which leads to pulse distortion, we consider the injection into the slab of Gaussian pulses of the form

$$
E_{\mathrm{inj}}=\sqrt{\frac{I_{p}}{\epsilon_{0} c S_{m}}} \exp \left[-t^{2} /\left(4 \sigma^{2}\right)-i \omega_{\mathrm{inj}} t\right],
$$

where $I_{p}$ represents the peak power, the corresponding pulse energy being $E_{p}=\sqrt{2 \pi} \sigma I_{p}$. The length of the simulations is $31 \mathrm{ps}$, i.e., $10^{3}$ steps. The pulse energy is kept as low as possible in order to see only the linear spectral filtering $\left(E_{p}=0.01 \mathrm{fJ}\right)$. In other words, the carrier density $N(z, t)$ remains close to zero along the slab during the pulse propagation. The carrier frequency of the pulse $\omega_{\text {inj }}$ is in all cases taken to be zero, meaning that the spectrum of the pulse is centered at the band edge of the QW absorber.

Figure 6 shows the optical spectra of the input (black solid lines) and the output (red dashed lines) pulses whose full-width at half-maximum (FWHM) is 800 fs. The bluest frequency components are strongly damped as intuition suggests. This effect would be obviously larger for the shorter pulses, which

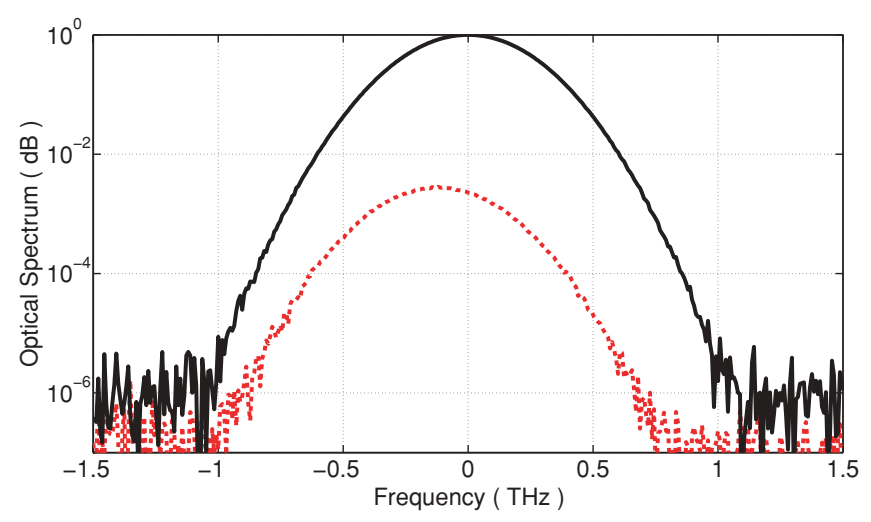

FIG. 6. (Color online) Optical spectrum of a 800 fs FWHM gaussian pulse after single propagation in the slab without QW (black line) and with QW (red dashes). For clarity, both spectra are normalized to the maximal spectral density of the pulse obtained without QW. 


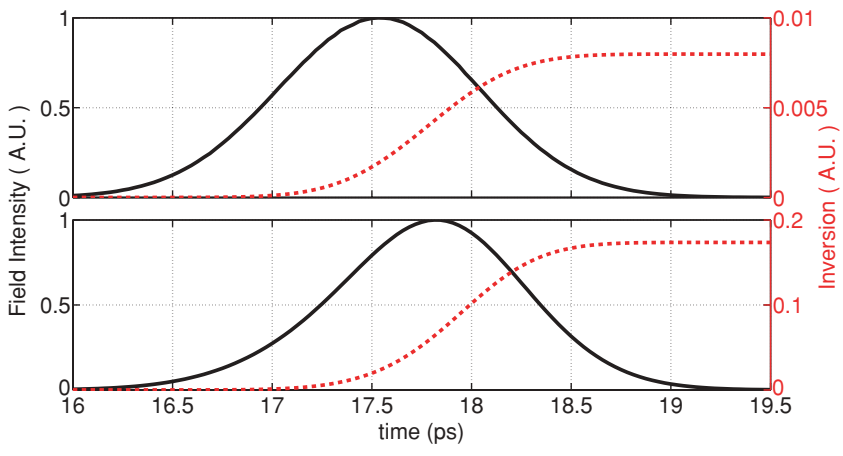

FIG. 7. (Color online) Time domain intensity profile of a $1 \mathrm{ps}$ FWHM pulse exiting the saturable absorber (black line) and carrier density at the output facet (red dots). From top to bottom, the pulse energy is $e=5.5 \mathrm{fJ}$ and $e=55 \mathrm{fJ}$, respectively. The pulse peak powers are normalized to unity for clarity. Notice that only the low energy pulse remains symmetric and that the more energetic the pulse, the longer the propagation time of the peak. On the bottom panel, substantial absorption occurs that significantly increases the carrier density close to the output facet.

span a larger frequency interval, but it is already clearly noticeable for $800 \mathrm{ps}$ pulses, hereby indicating the need to consider absorption dispersion when studying systems with a saturable absorbers as, e.g., passively mode-locked lasers. Clearly, after a single propagation trough the slab, the output pulses in time domain are distorted, but more important, they present strong chirping due to this linear spectral filtering effect.

\section{Large signal multimode response}

Finally, in order to study saturable absorption effects as the pulse propagates in the QW system, we consider injected pulses of constant FWHM of 1 ps but of increasing energy. The black lines in Fig. 7 show the output pulses (normalized to a maximum power of unity for the sake of comparison) obtained for energies $E_{p}=5.5 \mathrm{fJ}$ (top) and $E_{p}=55 \mathrm{fJ}$ (bottom). The corresponding to peak powers are $I_{p}=2.2 \mathrm{~mW}$ and $I_{p}=$ $22 \mathrm{~mW}$, respectively, well below the saturating power $I_{\text {sat }}$. In addition, the red dots depict the carrier density at the output facet obtained in each case.
We can clearly observe that only the low energy pulse keeps an almost symmetric intensity profile since the carrier density is always kept at a low level. Instead, for intense pulses, the leading edge is absorbed and the resulting pulse shape becomes quite asymmetric. In this case we can also observe that the carrier density at the output facet is substantially different from zero, indicating the presence of strong saturation in the absorption of the QW. We can moreover observe that the peak of the pulse exits the slab after a propagation delay that increases with the pulse energy. This is not only a consequence of the pulse distortion due to the absorption, but also due to the increase in effective group index associated with carrier generation.

\section{CONCLUSIONS}

We have presented a time-domain analytical description of the optical response of quantum well within the quasiequilibrium approximation valid for nonsaturating fields. The convolution approach adopted here allows to treat systems which are beyond reach of the Padé approximation developed in [36]. We have thoroughly analyzed the numerical implementation of the method and the potential sources of error. We have shown that the method can be numerically implemented both accurately and efficiently by artificially reducing the absorption band of the QW medium without sacrificing the fidelity of the resulting dynamics. The method has been applied to the analysis of pulse propagation in a waveguide with an unpumped QW in its core that acts as a saturable absorber. The obtained results are in good correspondence with the reported behavior of these systems, and build confidence in the use of the method as an efficient and accurate tool for modeling the time response of QW media.

\section{ACKNOWLEDGMENTS}

J.J. acknowledges useful discussions with J. M. Arnold and A. Pérez-Serrano and the financial support from EPSRC (project EP/E065112/1-High Power, High Frequency Modelocked Semiconductor Lasers). S.B. acknowledges financial support from project BLANCO (PN TEC2006-13887C05-03).
[1] I. I. Rabi, Phys. Rev. 51, 652 (1937).

[2] J. H. Van Vleck and V. F. Weisskopf, Rev. Mod. Phys. 17, 227 (1945).

[3] R. Karplus and J. Schwinger, Phys. Rev. 73, 1020 (1948).

[4] S. H. Autler and C. H. Townes, Phys. Rev. 100, 703 (1955).

[5] M. C. Newstein, Phys. Rev. 167, 89 (1968).

[6] R. J. Glauber, Phys. Rev. 131, 2766 (1963).

[7] A. E. Siegman, Lasers (University Science Books, Mill Valley, CA, 1986).

[8] K. Shimoda, Introduction to Laser Physics, Springer Series in Optical Sciences 44 (Springer-Verlag, Berlin, 1986).

[9] H. Risken and K. Nummedal, J. Appl. Phys. 39, 4662 (1968).

[10] R. Graham and H. Haken, Z. Phys. A 213, 420 (1968).
[11] S. Haroche and F. Hartmann, Phys. Rev. A 6, 1280 (1972).

[12] J. Javaloyes, M. Perrin, and A. Politi, Phys. Rev. E 78, 011108 (2008).

[13] G. P. Agrawal and N. K. Dutta, Long-Wavelength Semiconductor Lasers (Van Nostrand-Reinhold, New York, 1986).

[14] B. Mroziewicz, M. Bugajski, and W. Nakwaski, Physics of Semiconductor Lasers (North-Holland, Amsterdam, 1991).

[15] L. A. Coldren and S. W. Corzine, Diode Lasers and Photonic Integrated Circuits (Wiley, New York, 1995).

[16] H. Casey and M. Panish, Heterostructure Lasers (Academic, New York, 1978).

[17] C. Henry, IEEE J. Quantum Electron. 18, 259 (1982). 
[18] D. Lenstra, B. Verbeek, and A. Den Boef, IEEE J. Quantum Electron. 21, 674 (1985).

[19] W. W. Chow, S. W. Koch, and M. Sargent III, SemiconductorLaser Physics (Springer-Verlag, Berlin, 1994).

[20] H. Haug and S. Schmitt-Rink, Prog. Quantum Electron. 9, 3 (1984).

[21] M. Lindberg and S. W. Koch, Phys. Rev. B 38, 3342 (1988).

[22] O. Hess and T. Kuhn, Phys. Rev. A 54, 3347 (1996).

[23] O. Hess and T. Kuhn, Phys. Rev. A 54, 3360 (1996).

[24] P. Enders, IEEE J. Quantum Electron. 33, 580 (1997).

[25] J. Hader, J. Moloney, and S. Koch, IEEE J. Quantum Electron. 35, 1878 (1999).

[26] S. Balle, Opt. Commun. 119, 227 (1995).

[27] J. Yao, G. P. Agrawal, P. Gallion, and C. M. Bowden, Opt. Commun. 119, 246 (1995).

[28] S. Balle, Opt. Lett. 27, 1923 (2002).

[29] S. Balle, Phys. Rev. A 57, 1304 (1998).

[30] J. Mulet and S. Balle, IEEE J. Quantum Electron. 38, 291 (2002).

[31] J. Mulet and S. Balle, Phys. Rev. A 66, 053802 (2002).

[32] J. Mulet and S. Balle, IEEE J. Quantum Electron. 41, 1148 (2005).

[33] J. Mulet and J. Mørk, SPIE 5452, 571 (2004).

[34] A. M. Yacomotti, L. Furfaro, X. Hachair, F. Pedaci, M. Giudici, J. Tredicce, J. Javaloyes, S. Balle, E. A. Viktorov, and P. Mandel, Phys. Rev. A 69, 053816 (2004).

[35] L. Furfaro, F. Pedaci, J. Javaloyes, X. Hachair, M. Giudici, S. Balle, and J. Tredicce, IEEE J. Quantum Electron. 41, 609 (2005).
[36] J. Javaloyes and S. Balle, IEEE J. Quantum Electron. 45, 431 (2009).

[37] C. Ning, R. Indik, and J. Moloney, IEEE J. Quantum Electron. 33, 1543 (1997).

[38] E. A. Avrutin, J. H. Marsh, and E. L. Portnoi, IEE Proc.Optoelectron. 147, 251 (2000).

[39] H. A. Haus, IEEE J. Sel. Top. Quantum Electron. 6, 1173 (2000).

[40] H. Haug and S. W. Koch, Quantum Theory of the Optical and Electronic Properties of Semiconductors (World Scientific, Singapore, 1993).

[41] P. L. Bhatnagar, E. P. Gross, and M. Krook, Phys. Rev. 94, 511 (1954).

[42] M. A. Bernard and G. Duraffourg, Phys. Status Solidi 1, 699 (1961).

[43] J. A. Fleck, Phys. Rev. Lett. 21, 131 (1968).

[44] K. Yee, IEEE Trans. Antennas Propag. 14, 302 (1966).

[45] J. Javaloyes and S. Balle, IEEE J. Quantum Electron. 46, 1023 (2010).

[46] L. Narducci and N. B. Abraham, Laser Physics and Laser Instabilities (World Scientific, Singapore, 1988).

[47] R. Courant, K. Friedrichs, and H. Lewy, Mathematische Annalen 100, 32 (1928)

[48] A. Pérez-Serrano, J. Javaloyes, and S. Balle, Phys. Rev. A 81, 043817 (2010).

[49] W. H. Press, S. A. Teukolsky, W. T. Vetterling, and B. P. Flannery, Numerical Recipes: The Art of Scientific Computing (Cambridge University Press, Cambridge, 2007).

[50] M. Matsumoto and T. Nishimura, ACM Transactions on Modeling and Computer Simulation 8, 3 (1998). 